\title{
AGILE TRANSFORMATION IN CLINICAL RESEARCH
}

\author{
Katarina B. Pavlović, Ivana Berić, Ljiljana Berezljev \\ Faculty for Project and Innovation Management, Educons University, Serbia
}

\begin{abstract}
We need well designed clinical trials in order to manage different health issues and deliver quality vaccines and drugs to patients. The whole process starts with the phase I of the trial, followed by phases II and III, after which the documentation is presented to the regulatory body, which reviews the data presented, going into the phase IV if required. The process ends with a license to market being issued. Trials are expensive, time consuming and with uncertain outcomes. The old ways of managing clinical trials do not give satisfactory results any more. It seems that the change is needed more than ever before. Clinical research organizations that manage clinical trials, being a part of a highly innovative industry, need innovation themselves. They need to adapt in order to stay competitive. In this paper, it is presented, according to the literature review, that a possible way to undergo adaptation is through agile transformation.
\end{abstract}

Key words: Adaptive clinical trials, agile transformation.

\section{INTRODUCTION}

Clinical trials are used to determine whether or not a new drug or a vaccine, developed by a pharmaceutical company, is both safe and effective for patients. It is of great importance that patients get new substances as soon as possible in order to treat illnesses. This is not possible however, because not only the process of drug development takes a very long time, but clinical trials which represent next step in the process, require more time before the product is brought to market.

Clinical trials are a part of the industry, in which constant pressure is put on cutting the costs, improving the quality, complying with the regulatory requirements and satisfying the needs of end users. Considering the complex and changing environment it is very difficult for research organizations (CRO) to keep up, adapt and stay competitive.

Professionals working in clinical trials, for this reason, face numerous challenges, among which the three dominant being trial designs $(21 \%)$, regulations $(15 \%)$ and costs $(15 \%)$, followed by patient access (12\%), staff (9\%) and technology (9\%). In Figure 1, six challenges are presented making $81 \%$ in total. (internet, retrieved on Nov $2^{\text {nd }}, 2018$ )

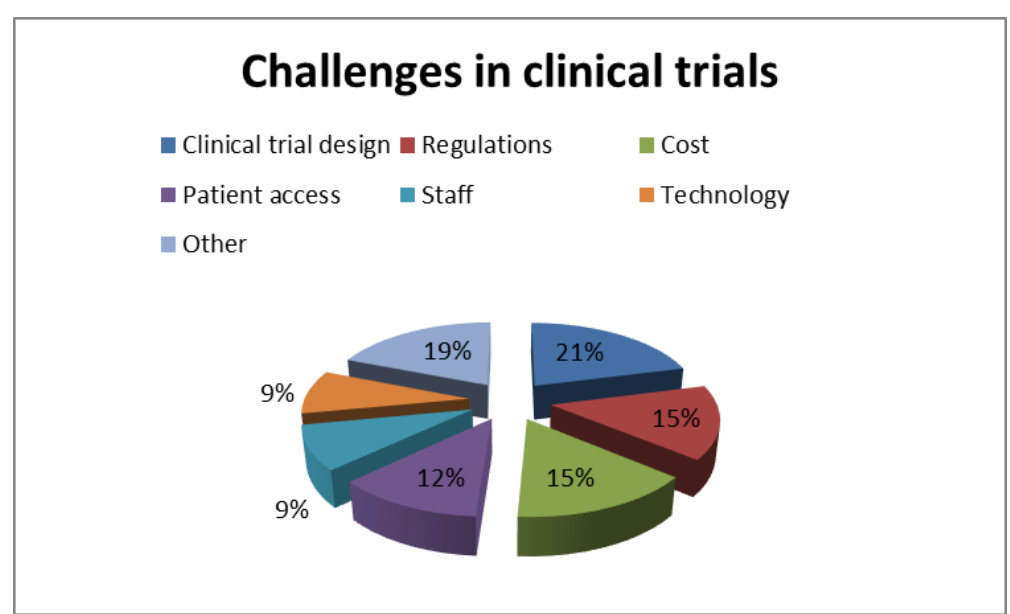

Figure 1: Challenges in clinical trials

Corresponding author. Email: kpavlovic25@gmail.com

ISSN 2560-4961(online)

(C) 2018 IPMA Serbia

doi: 10.18485/epmj.2018.8.1.8 
In the past twenty five years, effort has been put in "adapting" clinical trials to challenging expectations, but in the past ten with more success. The model suggested by regulatory bodies was "adaptive clinical trial design" or "adaptive clinical trials". This was a totally new approach in the pharmaceutical industry and it has been used ever since. (Nelson, 2010) It has been shown that the use of adaptive design shortens product development time and is therefore very cost-effective for industry. (Mehta, et al., 2009) However, even today it does not exceed $10 \%$ of all trials, both traditional and adaptive.

"Adaptive clinical trial design" is defined as a design that allows modifications to the trial and statistical procedures of the trial after its initiation, without undermining its validity and integrity. The role of adaptive design is to make clinical trials more flexible, more efficient and faster. Because of the level of flexibility involved, these trial designs are also termed as flexible designs. In essence a clinical trial that uses "adaptive design" allows, and indeed plans for, substantial change as the trial progresses without the need for a separate new study or a protocol amendment. (JahnEimermacher, \& Ingel, 2009)

It would be expected that "adaptive clinical trials" take more than $10 \%$ of all trials.

The answer may be in the fact that design methods are not yet clear enough and that this keeps sponsors, investigators and reviewers away from this approach.

In this paper a suggestion is made that one possibility is to switch to "adaptive clinical trials" through agile transformation, which would make those "agile clinical trials".

Clinical trials face numerous challenges, among which the three dominant being trial design $(21 \%)$, regulations (15\%) and costs $(15 \%)$. In picture 1 , six challenges altogether are presented making $81 \%$ in total and according to the statistical data. (internet, retrieved on Nov $2^{\text {nd }}, 2018$ )

\section{AGILE TRANSFORMATION}

In the demanding and fast changing environments of today's organizations, it seems that agile transformation is a possible choice for success. It has been present in the last two decades, starting with the software development and moving across other industries as well.

If the organization has been traditionally managed, this transformation will include radical shifts in attitudes, values, mindsets, ways of thinking and ways of interacting with the world, in effect, a change in organizational culture.

In companies where project management methodologies are applied, a transition process from traditional project methodologies to agile project methodologies is known as an agile transformation process and it involves almost all areas of organization. (Gandomani, \& Nafchi, 2015) It can be said that the main goal of the agile transformation is to provide organization with an agility feature. Organizational agility implies the ability to respond rapidly, proactively and intentionally to unexpected changing demand, while controlling the risk, adapting quickly and efficiently and innovating. (Appelbaum, Calla, Desautels, \& Hasan, 2017) Agility enables organization to acquire the knowledge, which will later apply to high quality products and services and also to coming up with responses to competitor's movements. (Cegarra-Navarro, Soto-Acosta, \& Wensley, 2016) The research results showed that a change of the project management methodologies may lead to wide, integrated and complex organizational changes in technology, methodology, processes, strategy and organizational culture increasing competitive advantage of the organization.

Agile methodology first started in 2001, as a set of principles for software development with the idea to write and release code iteratively without waiting for months to release functionality. The term "agile" is now shifting to organizations wishing to develop products or services iteratively, release frequently, keep focus on the customer and collaborate in a cross functional team, always emphasizing 
"test and learn" methods over detailed planning.

Agile, fundamentally is a redesign of the operating model of the enterprise.

Many of the world's biggest companies are struggle in their operating mode which is outdated and which makes them less productive than they should be. (Semolič, Jovanović, Obradović, \& Kovačev, 2008) What holds them back is a traditional business model that has been created for scale and standardization, rather than for agility and innovation.

Coming from software development agile transformation is not just about technology. It is about a new way of thinking. It is a more collaborative, more open, more creative and much more efficient than other business models. (Bogojević, 2017) It is something that can be implemented across the company, not just one or two departments. Companies can achieve agile transformation at three levels: the project level, which is easier to accomplish, the portfolio level, which is more complex and the organization level, which requires a complete rethinking of a company's operating model. It is not easy to move effectively from the first level to the last, but moving in progressive steps can be successful.

Clinical research organizations could also benefit from this kind of transformation. It would help in moving to "adaptive clinical trial design" and it would help in making the whole organization more flexible, adaptable and being able to respond to change.

\section{AGILE TRANSFORMATION CLINICAL RESEARCH}

IN

If we go in further details, in clinical research project management agile transformation is something that can help face most of the challenges presented in Picture 1 of this paper, where trial design is the first and the biggest one. It will be shown in the following discussion that regulatory bodies also call for a change in a trial design, suggesting "adaptive" design. This means that agile transformation is a broader approach and not only will requirements for "adaptive" trial design be fulfilled but many advantages will be brought to CROs such as:

- Being adaptable and being able to foresee future needs

- Improvements in cutting costs

- Lowering the amount of documentation through digital transformation

- Boosting creativity

- Finding better solutions and looking for innovations

Is it possible to consider going from "adaptive clinical trials" to "agile clinical trials"?

Adaptive clinical trials by definition allow for change during the trial without loss of validity. Although this stands to bring many advantages to enhancing research in resource-limited setting, there are limitations and potential pitfalls. Less familiar design methods incorporating these methodological features might be putting off for sponsors, investigators, and reviewers. However, experience is growing and more reports using these methods are becoming available.

Adaptive trial designs have been in use for the past 25 years, but gain more attention in the past 10 years. Both European and American regulatory bodies strongly encourage adaptive trial design. Following a workshop at the European Medicines Agency (EMA) involving academia, industry and regulators in 2007, the Reflection Paper on Methodological Issues in Confirmatory Clinical Trials Planned with an Adaptive Design was issued. (EMA, 2007). The FDA guidance document, from 2010, provides detailed notes on how risks to study validity and interpretation can be avoided and these could reassure and help guide investigators and statisticians. (FDA, 2010). It states that "There is great interest in the possibility that clinical trials can be designed with adaptive features (i.e., changes in design or analyses guided by examination of the accumulated data at an interim point in the trial) that may make the studies more efficient (e.g., shorter duration, fewer patients), and more likely to demonstrate an effect of the drug if one exists, or more informative (e.g., by providing broader dose-response information)". (FDA, 2010)

In late 2016, the US Congress passed into law the 21st Century Cures Act, which instructs the 
Food and Drug Administration (FDA) to update its guidance on adaptive designs for sponsors of investigational drugs and biological products. The legislation refers to adaptive designs as "modern" and "novel" methods. (US Gov., 2016)

It is expected that EMA will provide more information on adaptive trials in new CTR waiting to be published in 2019 .

Some adaptive methods are indeed recent developments, while others have existed for decades and have had a complex history.

In no sense should adaptive design be seen as a shortcut or less rigorous approach, rather as an optimal strategy that allows for a balance between learning about an unknown effect to benefit future patients and the ethical obligation to provide the best treatment of the patient on the trial. Indeed, it is unlikely that industry would have succeeded in widespread use of a design approach that risked suggestion of seeking shortcuts and weaker trial data. (Chin, 2012)

How can adaptive design in clinical trials be connected with agile methodologies in clinical trials?

Since agile methodologies were primarily used for software development, it seems that there is no real connection between IT and pharmaceutical industry, unless agile methodology is used for software development in clinical research. This changes when we look at the medicine as a software and try to consider the possibility that a software continues to evolve while it is used by patients. There are two possibilities: the software is going to be released faster with very little scientific evidence or we are going to release an obsolete software in order to wait for all of the scientific studies to be completed.

Experiences in different CROs show that the agile approach is applicable and that it gives results. Considering constant changes and unknowns in the future, it is not possible to foresee for how long it will last, but it is promising at the moment. Agile methodologies brought many positive aspects to CROs, such as:
- Statistical data show that the duration of studies was cut by $30-40 \%$.

- Time to market was shortened (exact data unavailable)

- Access to patients and selection process were optimized

- The quality of product was raised if agile transformation is applied throughout the entire process of drug development

- Competitive advantage was gained

\section{APPROACH AND OBSTACLES TO IMPLEMENTATION}

Literature shows that only $10 \%$ of CROs use "adaptive" or "agile" approach, which indicates that they encountered many obstacles along the way. The most important ones are: Lacking management support and understanding, uncertainty regarding the outcomes, unclear choice of methods, costs etc.

Research results and conclusions regarding agile transformation should be used by CROs' management in order to facilitate this process with less cost, time and effort. Every new approach brings uncertainty and without uncertainty, there is no innovation. In today's world, we need to embrace uncertainty. Many argue that most methods used in IT sector are impossible to apply to clinical research, thinking primarily of Scrum as a method of choice. This can be argued in different ways. It is obvious that Scrum implies small teams working together, no influence on the design process, very little if none external control, Product owner (in the case of clinical trials Sponsor) being involved in certain fazes only and according to many Scrum is not to be used in the development of products with critical application (air, automotive and pharmaceutical industry). These arguments are correct, but only if we forget that agile transformation is about a different mindset, about a new way of thinking and acting and not about a strict application of a method such as Scrum or any other. As the cost of "agile" transformation in terms of money, disrupted working routines and quality of development may become fairly significant, there is a need to quantitatively measure the impact of an agile transition (Olszewska (née Pląska), Heidenberg, Weijola, Mikkonen, \& Porres, 2016). 
The solution for this complex problem is that CROs need to develop their own combination of methods, relying on competent, motivated and trained people who will lead their organizations through "agile" transformation, understanding that this is the way to deliver their customers high quality products, faster and at lower costs.

\section{CONCLUSION}

In this paper it was shown that an agile transformation and application of agile methodologies in clinical research project management can shift clinical trials from "standard" to "adaptive" and "agile" approach. Agile transformation in clinical research project management, fulfills the need to make clinical trials "adaptive", and at the same time offers a broader perspective. It is a complete organizational makeover, but one of the best options available. The process is long, expensive and demanding. While regulators call for adaptive clinical trials, CROs could move one step ahead and go with agile clinical trials. This is not an easy path to follow and a lot of effort needs to be made in order to achieve the goals. This would bring several advantages to pharmaceutical drug development, such as bringing new drugs and vaccines to patients faster, cutting costs, improved patients' selection, more creativity and innovation and a lot more. Since it is indicated that this approach comes with a support from FDA and EMA, in the form of adaptive trials, these novel trials will bring new aspect of quality to pharmaceutical companies.

What seems to have been an obstacle in implementation so far is the choice of design methods. CROs have been struggling to find the best solutions and currently it is done through "in house best practice". This is because agile methodology is not applicable neither to all parts of a study nor to all kinds of studies, but striving for an agile transformation in an organization as a whole will pave the way in making clinical trials as "adaptive" as possible. It is certain that each organization does need its own methods which "work the best", but it is also recommended to exchange the experiences more in order to go through this transformation faster.

\section{REFERENCES}

Appelbaum, S. H., Calla, R., Desautels, D., \& Hasan, L. (2017). The challenges of organizational agility (part 1). Industrial and Commercial Training, 49(1), pp. 614.

Bogojević, P. (2017). Comparative analysis of agile methods for managing software projects. European Project Management Journal, Vol 7, Issue 1, pp. 58-74.

Cegarra-Navarro, J. G., Soto-Acosta, P., \& Wensley, A. K. P. (2016). Structured knowledge processes and firm performance: The role of organizational agility. Journal of Business Research, 69(5), pp. 1544-1549.

Chin, R. Y. (2012). Adaptive and flexible clinical trials. Boca Raton: CRC Press

European Medicines Agency. (2007). Reflection Paper on Methodological Issues in Confirmatory Clinical Trials Planned with an Adaptive Design (CHMP/EWP/2459/02)

FDA. (2010). Guidance for Industry: Adaptive Design for Drugs and Biologicals. 2010.

Gandomani, T. J., \& Nafchi, M. Z. (2015). An empirically-developed framework for Agile transition and adoption: A Grounded Theory approach. The Journal of Systems and Software, 107(9), pp. 204219.

https://knect365.com/clinical-trialsinnovation/article/, retrieved on Nov $2^{\text {nd }}$, 2018.

https://knect365.com/clinical-trialsinnovation/article/, retrieved on Nov $2^{\text {nd }}$, 2018.

Jahn-Eimermacher, A., \& Ingel, K. (2009). Adaptive trial design: a general methodology for censored time to event data. Contemp Clin Trials. 30, pp. 171177.

Mehta, C., Gao, P., Bhatt, D. L., Harrington, R. A., Skerjanec, S., \& Ware, J. H. (2009). Optimizing trial design: sequential, adaptive, and enrichment strategies. Circulation. 119, pp. 597-605.

Nelson, N. J. (2010). Adaptive clinical trial design: Has its time come? J Natl Cancer Inst.102, pp. 1217-1218. 
Olszewska (née Pląska), M., Heidenberg, J., Weijola, M., Mikkonen, K., \& Porres, I. (2016). Quantitatively measuring a largescale agile transformation. Journal of Systems and Software, 117(7), pp. 258273

Semolič, B., Jovanović, P., Obradović, V., \& Kovačev, S. (2008). Improving repair management of bucket wheel excavator SRs1200 by application of project management concept. Strojniški vestnik -
Journal of Mechanical Engineering, 54(78), pp. 565-573.

United States Government. Rules committee print 114-67, text of House amendment to the Senate, amendment to H.R. 34, Tsunami Warning, Education, and Research Act of 2015, 2016:162-3. 114th Congress http://docs.house.gov/billsthisw eek/20161128/CPRT-114-HPRT-RU00SAHR34.pdf 